\title{
Relays-Enhanced LTE-Advanced Networks Performance Studies
}

\author{
Mattia Minelli*, Marceau Coupechoux ${ }^{\dagger}$, Jean-Marc Kelif ${ }^{\ddagger}$, Maode Ma* and Philippe Godlewski ${ }^{\dagger}$ \\ *Nanyang Technological University \\ Singapore \\ mattia1@e.ntu.edu.sg, \\ emdma@ntu.edu.sg \\ ${ }^{\dagger}$ Telecom ParisTech - CNRS LTCI \\ Paris, France \\ \{coupecho,godlewsk\}@enst.fr
}

\begin{abstract}
This paper proposes a study on the performance of multi-hop relays networks. We demonstrate the importance of the relays for coverage and capacity enhancement and study how the number of relays and their position inside the cell impact the performance, and how an efficient relay location scheme can guarantee better performance with less relays per cell. Further we give an estimation of the amount of power per square meter on the ground when relays are used, with respect to traditional network, showing the fundamental role of relays for green networking. We consider the downlink of an LTE-Advanced network, in which all the User Equipments (UE) are supposed to be attached to the eNode-B (eNB) or Relay Node (RN) from which they receive more power. Numerical results are obtained through Monte-Carlo simulations and the performance measures are represented by the average effective Signal to Interference and Noise Ratio (SINR) and the average spectral efficiency.
\end{abstract}

\section{INTRODUCTION}

The growing demand for mobile internet and wireless multimedia applications and the ITU-R/IMT advanced demanding requirements for the future $4 \mathrm{G}$ systems have pushed the search for means to improve networks throughput and coverage. One of the most promising solutions is represented by Multi-hop Relay (MR) networks, which can guarantee better network performance at the price of an increased network complexity.

Relay Nodes (RNs) are defined [1] as devices which are wirelessly connected to the radio-access network via a donor cell; they communicate with both their controller eNBs and their controlled UEs, and perform a decode-and-forward operation on the processed signals (unlike RF repeaters, which perform an amplify-and-forward operation). Deployment of RNs gives the network a hierarchical structure, where the informations from and to UEs can be directly exchanged with the eNB or pass through an RN.

RNs can operate in simultaneous mode of operation, sharing the same time-frequency resources with their controller eNB, or in division mode of operation, using non-overlapping timefrequency resources. In this way, in-sector interference can be avoided. Division mode of operation can be achieved e.g. by mean of Time-Division Duplex (TDD) mode, in which eNB and RNs transmit in different time instant, or FrequencyDivision Duplex (FDD) mode, in which eNB and RNs transmit using multiple carrier frequencies.
Several papers have been produced so far about MR networks in an LTE-Advanced context to show either coverage or capacity gains. The study in [2] deals with network performance both on the uplink and on the downlink, assuming the use of a considerable number of RNs deployed near the cell edge. A strategy for power control optimization is introduced, and the concept of cell coverage extension using RNs is explained and validated through simulations. Coverage extension is also studied in [3]. Authors of this paper analyze the coverage gain that can be obtained by adding RNs, while considering a fixed capacity. The ratio of the number of eNB to the number of RNs is carefully studied.

The advantage of MR networks in terms of capacity is part of [4], which gives SINR vs mutual information curves, for different Modulation and Coding Schemes (MCS). An extensive description of the different relaying strategies is given in [5], which considers the downlink sum rate as main performance measure. Finally, the average SINR and data rate for all locations in the cell are given in [6], through a numerical study making use of a close SINR formula.

The aim of this paper is to give an overview of the relaysenhanced cellular networks downlink performance in an LTEadvanced context in terms of average capacity, average SINR and stations coverage. We also concentrate on the TDD frame design and propose a resource partition between eNode-B and RN. At last, we study the effect of RN deployment on the global energy consumption of the network. The study is performed for three specific deployment scenarios suggested by evaluation methodology documents.

The paper is organized as follows. Section II describes the network model we consider. Section III details our simulation results. At last, Section IV concludes the paper.

\section{Network AND InTERFEREnCE Model}

In this section, we describe the network and interference models and provide the effective SINR formulation.

\section{A. Network Model}

In this paper, we consider an LTE-Advanced hexagonal network, whose access infrastructure is made of a set $\mathcal{S}_{e N B}$ eNBs and a set $\mathcal{S}_{R N}$ of RNs and we study the performance of this cellular network on the downlink. eNBs have a cell range 
of $R$ and are supposed to be tri-sectored. Each sector contains one or several omni-directional RNs. UEs are supposed to be served by the station (eNB or $\mathrm{RN}$ ) from which they receive the highest power including shadowing (best server policy).

Throughout the paper, we assume that all eNBs on the one hand, all RNs on the other hand, transmit at the same power per sub-carrier: $\forall i \in \mathcal{S}_{e N B}, P_{t x, i}=P_{e N B}$ and $\forall i \in \mathcal{S}_{R N}, P_{t x, i}=P_{R N}$.

\section{B. Propagation}

Let us denote $P_{r x, i}(r, \theta)$ the power received by an UE, where $r$ is the distance between the UE and its serving station $i \in \mathcal{S}_{e N B} \cup \mathcal{S}_{R N}$ and $\theta$ represents the angle between the transmitting station-UE direction and the transmitting station antenna boresight direction. The received power can be written:

$$
P_{r x, i}(r, \theta)=P_{t x, i} K r^{-\eta} S A(\theta) \beta,
$$

where $K$ is a constant, $\eta$ is the path loss exponent, $S=10^{\frac{\xi}{10}}$ is a lognormal random variable (RV) taking into account the variations over the received power due to the shadowing effect, $\xi$ is a normal zero-mean $\mathrm{RV}$, whose standard deviation is denoted $\sigma$ (in $\mathrm{dB}$ ), $A(\theta)$ is the gain due to the transmitting station antenna pattern and $\beta$ is a RV taking into account the effect of the fast fading. Fast fading is drawn in agreement with the statistical features of the Typical Urban Macrocell Channel given in [7], as required in [1].

\section{Frame Structure}

In order to avoid inner sector interference we adopt a timedivision between eNB-RN, eNB-UE and RN-UE transmissions. The frame temporal structure used in the simulations will be of the kind depicted in Fig. 1, where the frame is divided into three parts. The first part of duration $\tau$ is dedicated to the eNB-RNs transmission, the second of duration $t_{e N B}$ is dedicated to the eNB-UE transmission and the third of duration $t_{R N}$ is dedicated to the RN-UE transmission.

In this paper, we will analyze to what extent relays are useful when $\tau$ is increasing. The case $\tau=0$ would correspond to the deployment of an optical fiber between eNB and RNs. The case $\tau>0$ corresponds to a RF communication between eNB and RNs. The higher is the capacity on this link, the smaller is $\tau$.

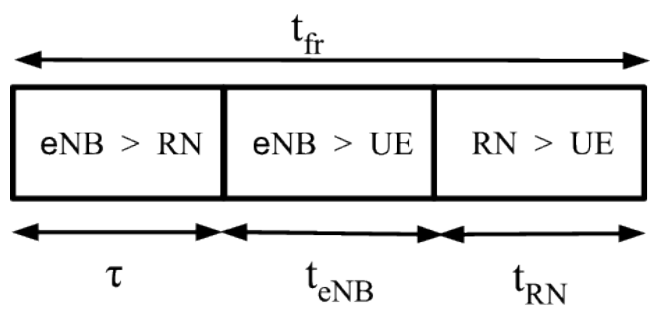

Fig. 1. Frame temporal structure.

\section{Relays Configurations}

Relays enhanced networks performances are obviously influenced by the number of RNs in each cell, and by the way the RNs are distributed inside the cell itself. In this paper we analyze and compare the performance of three possible RNs deployments.

1) Six Relays per Cell (6RPC) Scenario: This scenario is taken from [8], and makes use of six relays in each cell, two per sector. Prescriptions from [8] require an angle $\phi$ of 26 degrees between the sector antenna boresight direction and the eNB-RN direction, as shown in Fig. 2 (a). The distance $d_{R N}$ between the eNB and each $\mathrm{RN}$ is required to be equal to $3 / 8$ of the Inter-Site Distance (ISD) [8].

In order to avoid in-sector interference, only one device out of the 3 deployed in each sector of a 6RPC scenario cell is supposed to be active at a given time instant. This means that the third part of the frame depicted in Fig. 1 is further divided into 2 periods for this scenario. Each period is dedicated to the transmission of one of the RN, while the other is not transmitting.

2) Three Relays per Cell (3RPC) Scenario: In this scenario three relays are deployed at the edge of each cell, where the three sector antennas boresight direction lines cross the cell edge. The resulting deployment pattern is drawn in Fig. 2 (b). The number of relays per sector is one.

3) Two Relays per Cell (2RPC) Scenario: The 2RPC scenario makes use of only two relays per each cell, deployed on two opposite cell corners, giving the deployment pattern shown in Fig. 2 (c). We notice that one sector of the cell does not include any RN, hence during the RN-UE transmission subframe, no station will be active in this sector. A possible drawback of this configuration lies in the position of the RNs, which are far from the sector antennas boresight directions.

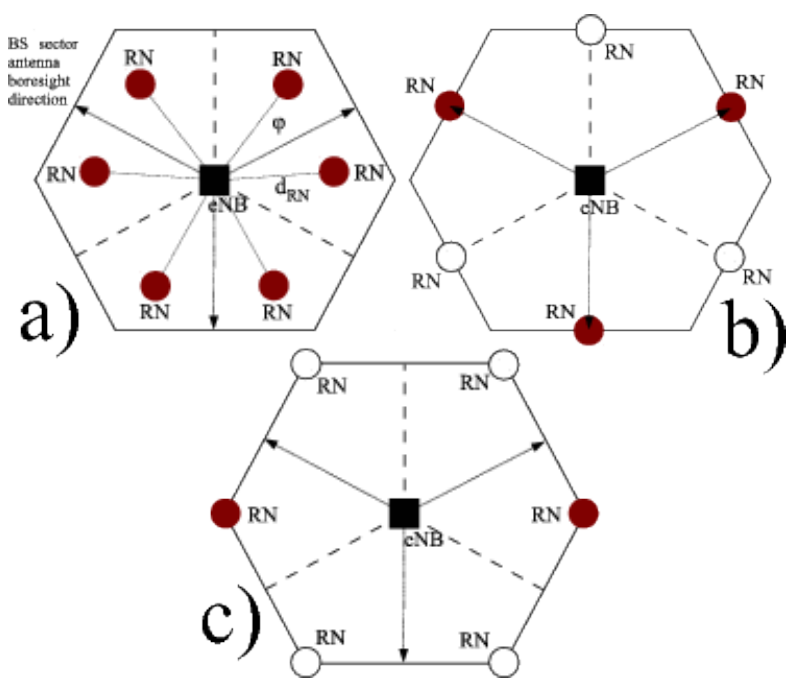

Fig. 2. a) 6RPC deployment b) 3RPC deployment. c) 2RPC deployment. White filled RNs indicate stations controlled by another eNB 


\section{E. SINR and Spectral Efficiency}

The SINR experienced by a UE on a given resource element (one OFDM symbol, one sub-carrier) is denoted by $\gamma_{s, c}$, where $s$ is the symbol index and $c$ the subcarrier index. It is computed as the ratio between the serving station received power and the sum of the interfering powers plus a background noise $N=N_{0} W_{c}$, where $N_{0}$ is the thermal noise spectral density $(-174 \mathrm{dBm} / \mathrm{Hz})$ and $W_{c}$ is the subcarrier bandwidth. If the serving station is indexed by $i, \gamma_{s, c}$ can be written:

$$
\gamma_{s, c}=\frac{P_{r x, i}}{\sum_{j \in \mathcal{S}, j \neq i} P_{r x, j}+N},
$$

where $\mathcal{S}$ is the set transmitting stations, $\mathcal{S}=\mathcal{S}_{e N B}$ or $\mathcal{S} \subseteq$ $\mathcal{S}_{R N}$ depending on the type of serving station (eNB or RN) and on the RN deployment scenario.

The basic allocation resource of LTE-Advanced is the Physical Resource Block (PRB), i.e., it is the smallest amount of resources which can be allocated by the eNB scheduler to a user, and its time-frequency dimensions are $N_{s}=6$ or 7 OFDM symbols (depending on whether the normal or extended cyclic prefix is used) by $N_{c}=12$ consecutive subcarriers [9]. We consider here the case $N_{s}=7$.

On a PRB, the amount of data is allocated according to the effective SINR $\Gamma_{e f f}$. It can be derived using the Mean Instantaneous Capacity (MIC) method [10]:

$$
\Gamma_{e f f}=2^{\sum_{s=1}^{N_{s}} \sum_{c=1}^{N_{c}} \log _{2}\left(1+\gamma_{s, c}\right) / N_{s} N_{c}}-1 .
$$

Average spectral efficiency obtained for a single PRB transmission during the RN-UE link period is now given by: $C_{R N}=E\left[\log _{2}\left(1+\Gamma_{\text {eff }}\right)\right][b p s / H z]$, where the expectation is taken over UEs attached to RNs. $C_{e N B}$ is defined in the same way for the eNB-UE link period, so that the overall spectral efficiency for a single PRB transmission is given by:

$$
C=p_{e N B} C_{e N B}+p_{R N} C_{R N},
$$

for scenarios with relays and by $\tilde{C}=C_{e N B}$ for scenarios without relays. $p_{e N B}$ and $p_{R N}$ denote respectively the probability for a UE to be attached to an eNB or an RN. Assuming fairness in the resources sharing between the UE, the average value of the total sector spectral efficiency depends on $\tau, t_{e N B}$ and $t_{R N}$, and it is given by

$$
C_{s e c t}=\frac{t_{e N B}}{t_{f r}} C_{e N B}+\frac{t_{R N}}{t_{f r}} C_{R N}
$$

for sectors where one $\mathrm{RN}$ is deployed, or one $\mathrm{RN}$ is active for the whole interval $t_{R N}$ (as in the 6RPC scheme). In the 2RPC scheme 2 sectors of the cell have a relay, while the third sector is covered by the eNB only. For this sector, the total spectral efficiency can be simply computed as $C_{e N B}$, and the average cell spectral efficiency can be derived as

$$
C_{s e c t, 2 R P C}=\frac{2}{3}\left(\frac{t_{e N B}}{t_{f r}} C_{e N B}+\frac{t_{R N}}{t_{f r}} C_{R N}\right)+\frac{1}{3} C_{e N B} .
$$

\section{Simulations Results}

In this section we show the numerical results obtained in Monte-Carlo simulations over relays-enhanced networks.

\section{A. Simulations Assumptions}

The semi-static two-dimensional simulations performed in this paper are compliant with [1]. The network cluster used to simulate a real network is formed by two cell rings around a central cell. Moreover, the wraparound technique has been employed (6 cell clusters 'wrapped' around the central cluster). All the SINR measurements have been carried out on UEs dropped uniformly in the central cluster (one UE per each sector, per each simulation snapshot). We assume that during each snapshot a PRB is transmitted contemporaneously by every network node included in the set $\mathcal{S}$ when the snapshot is taken (full load assumption).

Simulations take into account the effects of path loss, shadowing, fast fading and eNB sector antenna patterns. A frequency correlation factor $\rho=0.5$ for fast fading is considered, while no line-of-sight propagation between UEs and stations is supposed. The doppler effect originated by the movement of the UE is also considered in the computation of the fast fading. Simulation details are provided in Table (I).

TABLE I

SIMULATION DETAILS

\begin{tabular}{|l|l|}
\hline Requirement & Value \\
\hline \hline eNB Total Tx Power & $46 \mathrm{dBm}$ \\
\hline RN Total Tx Power & $30 \mathrm{dBm}$ \\
\hline Freq. reuse pattern & $1 \times 3 \times 1$ \\
\hline ISD & $0,5 \mathrm{Km}$ \\
\hline Propagation constant & $\eta=3.75$ \\
\hline Shadowing standard deviation & $\sigma=10 \mathrm{~dB}$ \\
\hline Antenna pattern $3 \mathrm{~dB}$ beamwidth $\left(\theta_{3 d B}\right)$ & $70^{\circ}$ \\
\hline Terminal speed & $3 \mathrm{Km} / \mathrm{h}$ \\
\hline Subcarrier bandwidth & $15 \mathrm{KHz}$ \\
\hline System bandwidth & $10 \mathrm{MHz}$ \\
\hline Simulation snapshots & 10000 \\
\hline
\end{tabular}

\section{B. SINR vs Distance}

Fig 3 plots the average SINR vs distance from the cell center, for the three introduced scenarios and allows a comparison of their performance. The average SINR at a given distance $d$ is defined as the average, computed over all the UEs at a distance $d$ from the cell center and all the snapshots, of the values of $\Gamma_{e f f}$ collected in simulations.

We notice how the performance improves while increasing the number of RNs in each cell, and how the shape of the curves is influenced by the deployment scenarios topologies. The high spike in the 2RPC scenario performance curve is due to the position of the RNs in this scenario: here RNs are deployed in the 'corners' of the cell, hence all the UEs at a distance close to the cell range $R$ from the cell center are very close to the RN. The average SINR for distances from cell center close to $R$ is thus computed on UEs which are very close to the RNs. 
The figure shows how the relays deployment can be useful to improve network capacity near the cell edge, which is one of the main reasons for RNs use in future networks.

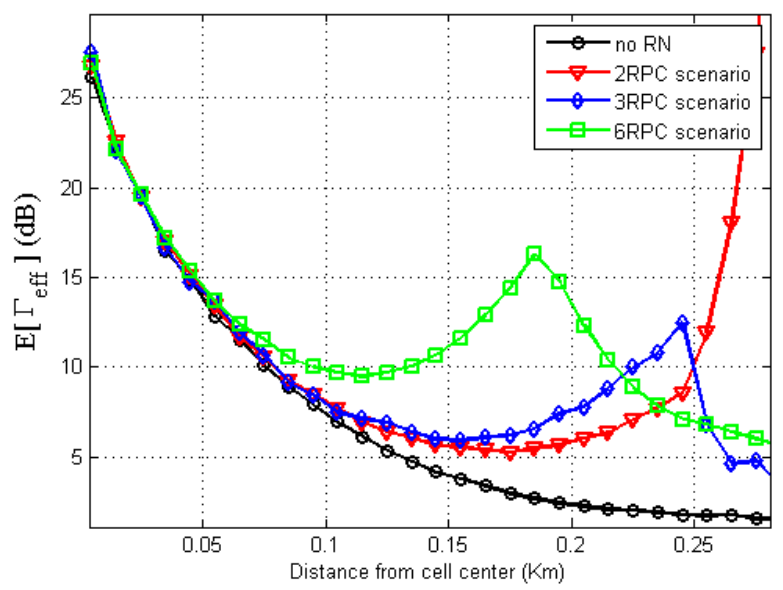

Fig. 3. Effective SINR vs distance from cell center: RNs deployment scenarios performance comparison.

\section{SINR CDF}

The average $\Gamma_{\text {eff }}$ Cumulative Distribution Function (CDF) in an RN enhanced cell is drawn in Fig. 4 for the 3 scenarios introduced, and compared with the case where no RNs are used. As we can see the use of a limited number of RNs (e.g. 2RPC) can sensibly boost the SINR. It is also interesting to notice how the 2RPC scenario has almost the same performance of the 3RPC scenario. This can be explained by the fact that in the 2RPC scenario the RNs deployed in each cell are less, but the RNs deployment scheme is more 'efficient' in terms of coverage (i.e. the RNs are placed in the farthest cell locations with respect to the eNBs).

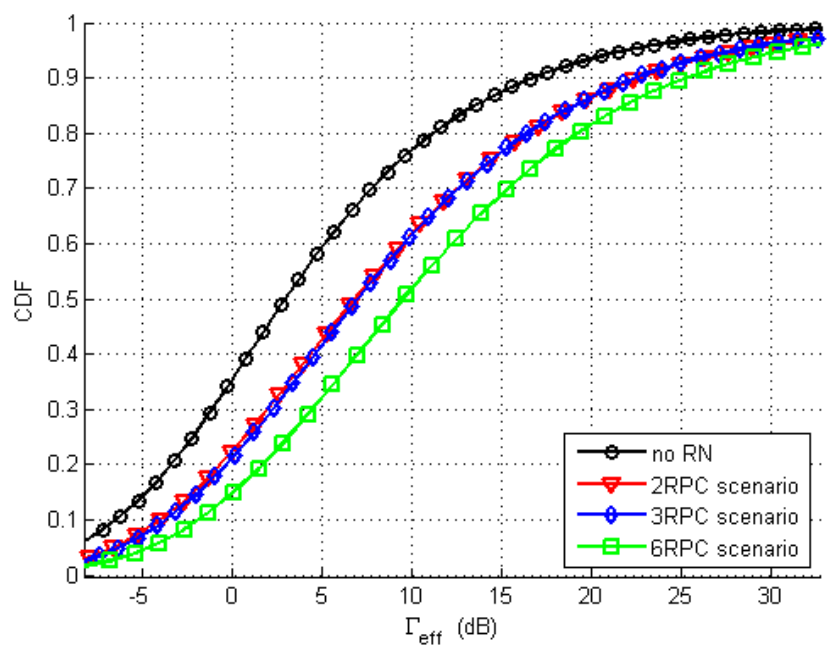

Fig. 4. $\Gamma_{\text {eff }}$ CDF: deployment scenarios comparison.

\section{D. eNB-RN Link Duration}

The performance of an MR cellular network is influenced by the quantity of resources dedicated to the eNB-RN transmission $\tau$. In this section we study the tradeoff between the gain given by the use of RNs in terms of instantaneous capacity and the average capacity loss given by the time dedicated to the eNB-RN link during the frame.

Let suppose we have an MR network using the frame structure of Fig. 1. Intuitively, the use of relays in the network is convenient only if $\tau$ is less than a certain threshold $\bar{\tau}$.

In order to find $\bar{\tau}$ we impose the average quantity of resources granted to the UEs served by an eNB on one subcarrier, named as $T_{e N B}$ and measured in bit/frame, to be equal to the average quantity of resources granted to the UEs served by an RN, and named as $T_{R N}$ :

$$
\begin{aligned}
T_{e N B} & =T_{R N} \\
\frac{C_{e N B} t_{e N B}}{p_{e N B}} & =\frac{C_{R N} t_{R N}}{p_{R N}},
\end{aligned}
$$

where $p_{e N B}$ and $p_{R N}$ denote respectively the probability for a UE to be served by an eNB and the probability for a UE to be served by an RN.

Taking into account that $t_{e N B}+t_{R N}=t_{f r}-\tau$ we obtain:

$$
t_{R N}=\frac{C_{e N B}\left(t_{f r}-\tau\right)}{C_{R N} p_{e N B}+C_{e N B} p_{R N}} p_{R N},
$$

and considering (8), we can rewrite the average quantity of resources $T$ granted to each $\mathrm{UE}$ as

$$
T=T_{e N B}=T_{R N}=\frac{C_{R N} C_{e N B}\left(t_{f r}-\tau\right)}{C_{R N} p_{e N B}+C_{e N B} p_{R N}} .
$$

The value of $\bar{\tau}$ is found by imposing $T$ to be equal to the quantity $\tilde{T}$ of resources granted to each UE when RNs are not deployed. Considering that $\tilde{T}=\tilde{C} t_{f r}$, we get

$$
\bar{\tau}=t_{f r}\left(1-\tilde{C}\left(\frac{p_{e N B}}{C_{e N B}}+\frac{p_{R N}}{C_{R N}}\right)\right) .
$$

Figure 5 shows proportion $\bar{\tau} / t_{f r}$ for the different relays configurations using (11). The values of $\tilde{C}, C_{R N}, C_{e N B}, p_{e N B}$ and $p_{R N}$ are obtained by mean of numerical simulations.

Analyzing Figure 5 we can see that in a 6RPC scenario we can dedicate more time (resources) to the eNB-RN link than in a $2 \mathrm{RPC}$ or $3 \mathrm{RPC}$ scenario, for a fixed capacity value.

\section{E. RN-eNB Relative Power Influence}

In this section we show the impact of a change of $P_{R N}$ on network perfomance. Fig. 6 depicts the $\mathrm{CDF}$ of $C$ for different values of $P_{R N}$, while $P_{e N B}$ is fixed. As expected, for low RNs transmitting powers the performance gets closer to the norelays case. Increasing RNs power more than a certain value doesn't bring any considerable advantage. 


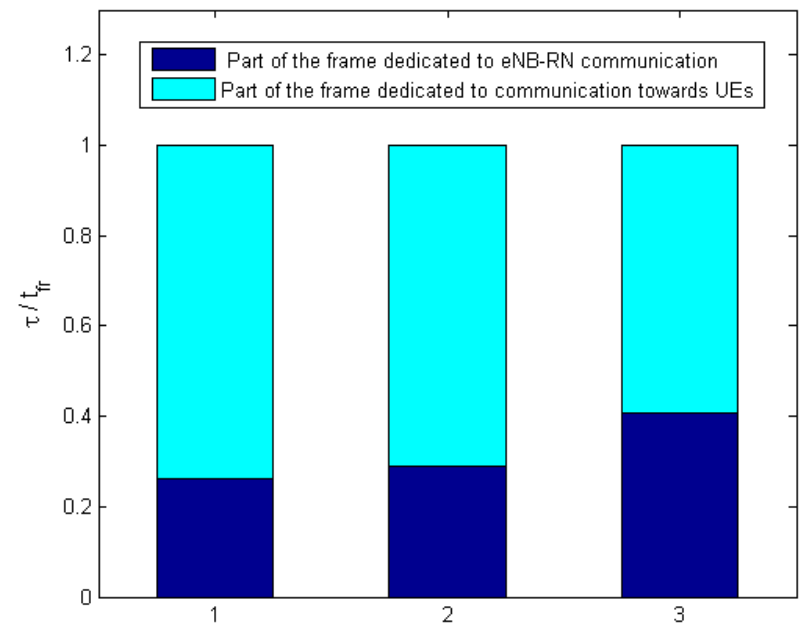

Fig. 5. Frame subdivision, for $\tau=\bar{\tau}$, with different RNs configuration. 1) 2RPC configuration; 2) 3RPC configuration; 3) 6RPC configuration

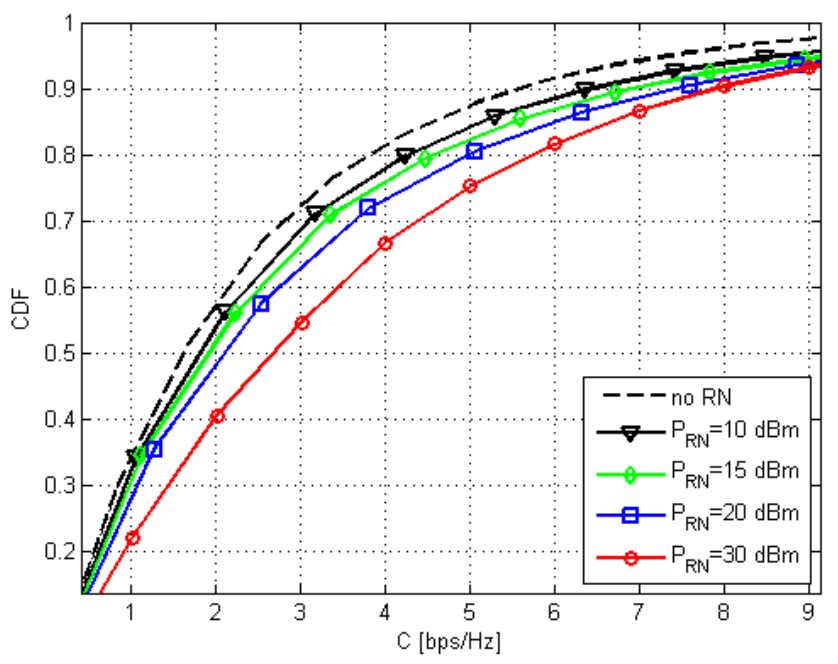

Fig. 6. CDF of $C$ for different values of $P_{R N}$ in a 2RPC scenario. Parameter $P_{e N B}$ is fixed to $46 \mathrm{dBm}$.

\section{F. Green Networking Using RNs}

An important advantage in the use of RNs lies in the possibility of lowering the average value of the surface power density $\delta$ on the cell, defined as the amount of received power per square meter, taking into account path loss and transmitting antenna pattern.

We propose here a comparative study about $E[\delta]$ in MR networks, assuming the eNB-RN communication to be performed with transmitted power $P_{e N B}$ and $\tau=\bar{\tau}$, in such way to keep the average capacity equal to the average capacity in a network without RNs. The average of $\delta$ over time and cell surface is given by

$$
E[\delta]=\frac{E\left[\delta_{e N B}\right] \bar{\tau}+E\left[\delta_{e N B}\right] t_{e N B}+E\left[\delta_{R N}\right] t_{R N}}{t_{f r}},
$$

where $\delta_{e N B}$ and $\delta_{R N}$ represent respectively the values of $\delta$ measured when eNBs or RNs are transmitting. Both $E\left[\delta_{e N B}\right]$ and $E\left[\delta_{R N}\right]$ can be obtained by simulations.

Defining $\delta$ analogously to $\delta$ for a network without RN, the value of the ratio $E[\delta] / E[\tilde{\delta}]$ is reported in Table II for the three RNs deployment scenarios introduced. The data in Table II demonstrate the consistent advantage in terms of surface power density given by MR networks.

TABLE II

VALUES OF THE RATIO $\delta / \tilde{\delta}$ FOR DIFFERENT RNS CONFIGURATIONS.

\begin{tabular}{|c|c|}
\hline Deployment scenario & $E[\delta] / E[\tilde{\delta}]$ \\
\hline \hline 2RPC scenario & 0.8437 \\
\hline 3RPC scenario & 0.7655 \\
\hline 6RPC scenario & 0.7011 \\
\hline
\end{tabular}

\section{CONCLUSION}

We have studied in this paper the effects of RNs deployment in an LTE-Advanced network, for three proposed deployment scenarios. We have seen how the use of RNs increases network capacity, and how this increase depends on both the number and the positions of RNs. Among the analyzed configuration scenarios the 2RPC can guarantee a good compromise between costs and capacity enhancement, while the 3RPC scenario appears to give no meaningful gains with respect to the 2RPC, and it employs more RNs per each cell. The 6RPC scenario gives the best performance, at the price of a greater costs compared to other scenarios. Network operators exigences and budget availabilities will determine the choice.

\section{REFERENCES}

[1] 3rd Generation Partnership Project (3GPP). TR 36.814 v9.0.0: Technical specification group radio access network; further advancements for EUTRA physical layer aspects (release 9), Sep. 2006.

[2] O. Bulakci and A. B. Saleh. Performance of relays in LTE-advanced networks. http://www.ikr.uni-stuttgart.de/Content/itg/fg524/Meetings/ 2010-02-18-Darmstadt/13_ITG524_Darmstadt_BouSalehBulakci.pdf, Feb. 2010.

[3] S. Redana J. Hamalainen T. Beniero and B. Raaf. Effect of relaying on coverage in 3GPP LTE-advanced. VTC09, Jul. 2009.

[4] R. Schoenen and B. H. Walke. On PHY and MAC performance of 3G-LTE in a multi-hop cellular environment. WiCom07, Sep. 2007.

[5] A. Y. Panah K. T. Truong S. W. Peters and R. W. Heath Jr. Relay architectures for 3GPP LTE-advanced. EURASIP Journal on Wireless Communications and Networking, 2009.

[6] R. Halfmann R. Schoenen and B. H. Walke. An FDD multihop cellular network for 3GPP-LTE. VTC08, May. 2008.

[7] IST-2003-507581 WINNER D5.4 v. 1.4. Final report on link level and system level channel models. http://www.ist-winner.org/ DeliverableDocuments/D5.4.pdf, Nov. 2005.

[8] R. Srinivasan J. Zhuang L. Jalloul R. Novak J. Park. IEEE $802.16 \mathrm{~m}$ evaluation methodology document (EMD). 2009.

[9] J. Zyren. Overview of the 3GPP long term evolution physical layer, Jul. 2007.

[10] K. Ramadas and R. Jain. WiMAX system evaluation methodology. Technical report, Jan. 2007. 